\title{
SUBARCSECOND-SCALE OPTICAL AND RADIO STRUCTURE CORRELATIONS IN SEYFERT GALAXIES
}

\author{
R.W. POGGE \\ Department of Astronomy, The Ohio State University, \\ Columbus, Ohio, USA \\ and \\ M.M. DEROBERTIS \\ Department of Physics \& Astronomy, York University, \\ North York, Ontario, Canada
}

\begin{abstract}
An unanswered question in the study of Seyferts is the nature of the relationship between the extended radio-continuum and optical emission-line gas. Detailed comparison is difficult as most Seyferts have radio structure on sub-arcsecond scales, while most optical imaging is on $1-2^{\prime \prime}$ scales. Despite this, some basic features have emerged. Extended radio and optical emission regions are generally aligned in projection, but the overall optical emission extends to much greater galactocentric radii. Pedlar et al. (1989) and Whittle et al. (1986) have reported a tendency for optical emission knots to lie behind the radio lobes, interpreted in terms of the "cooling length" of gas heated by a radiative bowshock driven into the ISM by a jet. In NGC 1068 (Cecil et al. 1990) and M51 (Cecil 1988), however, ambient gas appears to be piling up ahead of a radio lobe at the terminus of the radio jet.

We have obtained subarcsecond seeing CFHT and HST Archive emission-line imaging of Seyfert galaxies, allowing a fair comparison of the radio and optical maps at identical scales. The bright knots of optical emission-line gas are primarily further from the nucleus than the radio knots, with a few showing cospatial emission. This is consistent with the radiative shock picture (e.g., Wilson 1989). In one case, Mrk 573, we see a distinctive bow-shaped morphology in the [OIII] emission-line gas. We also see S-shaped distributions of emission-line knots on scales larger than the radio structure. Ionization cones, where present in these galaxies, appear as a diffuse background upon which the bright knots associated with the radio structures are superimposed.
\end{abstract}

\section{References}

Cecil, G., Bland, J., \& Tully, R.B. 1990, ApJ, 355, 70

Cecil, G. 1988, ApJ, 329, 38

Pedlar, A. et al. 1989, MNRAS, 238, 863

Whittle, M. et al. 1986, MNRAS, 222, 189

Wilson, A.S. 1989, in Extranuclear Activity in Galaxies (ESO Conference and Workshop Proceedings 32), ed. E.J.A. Meurs \& R.A.E. Fosbury, 215.

T. J.-L. Courvoisier and A. Blecha: Multi-Wavelength Continuum Emission of AGN, 455.

(C) 1994 IAU. Printed in the Netherlands. 